\title{
Performance analysis of Carbon Nano Tubes
}

\author{
P.S. Raja, R.joseph Daniel, Bino. N \\ Dept. of E \& I Engineering, Annamalai University, Annamalainagar, 608 002, Tamilnadu, India.
}

\begin{abstract}
Carbon nanotubes (CNTS) have received much attention for their unique characteristics as a possible alternative to $\mathrm{Cu}$ interconnect. $\mathrm{Cu}$ interconnect dimensions begin to come into the range of mean free path of electron typically 40nm. This results in surface and grain boundary scattering. Owing to these scattering phenomena resistivity of $\mathrm{Cu}$ begins to increase. This drives us to look for new materials for future very large scale integration (VLSI) interconnects. Carbon nanotubes are alternative to copper because of their remarkable conductive, mechanical and thermal properties. They are effectively long, thin cylinders of graphite which is made up of layers of carbon atoms arranged in a hexagonal lattice with stiffest and strongest fibers. Carbon nanotubes have superior properties like current carrying capacity and conductivity compared to $\mathrm{Cu}$ interconnect. It exhibits metallic or semi-conducting property depending on chirality.

A matlab coding has been developed to evaluate the performance of various carbon nano tubes (CNT). The various parameters evaluated are Resistance, Capacitance and Inductance. The evaluations of these parameters are very important since they affect the performance of the device.
\end{abstract}

\section{Introduction}

Carbon nanotubes are widely used in nanoelectronics because of its mechanical and electrical properties [1]. With one hundred times the tensile strength of steel, thermal conductivity better than all but the purest diamond, and electrical conductivity similar to copper, but with the ability to carry much higher currents, they seem to be a wonder material. Metallic carbon nanotubes have been identified as a possible interconnect material of the future technology generations and be heir to $\mathrm{Al}$ and $\mathrm{Cu}$ interconnects. It can be metallic or semiconducting depending upon chirality. It has a wide range of application in the fields like big markets, flat panel display, lighting, fuel cells, electronics, biosensors, electrostatic painting of composites in products (car parts). The changes in resistance and capacitance during interconnect scaling poses a serious challenge to the interconnect delay. Therefore the analysis of various parameters like Resistance, Capacitance and Inductance has a significant impact on the performance and reliability of VLSI circuits.

\section{Carbon nanotubes}

Carbon nanotubes were discovered in 1991 by SUMIO IIJIMA of NEC. Carbon nanotubes are alternative to copper because of their remarkable conductive, mechanical and thermal properties [2], [4]. They are effectively long, thin cylinders of graphite. Graphite is made up of layers of carbon atoms arranged in a hexagonal lattice, like chicken wire. Carbon nanotubes are molecular-scale tubes of graphitic carbon with stiffest and strongest fibres. With one hundred times the tensile strength of steel, thermal conductivity better than all but the purest diamond, and electrical conductivity similar to copper, but with the ability to carry much higher currents, they seem to be a wonder material. There are six types of carbon nanotubes, of which mostly used single-walled and Multi-walled nanotubes. They are Long, Short, Single-walled, Multi-walled, Open and Closed types.

The structure of carbon nanotubes consist of one or more coaxial cylindrical sheets of graphite with an aspect ratio typically greater than 100 and outer diameter measuring tens of nanometer and closed at ends with two semi domes. Essentially two families of carbon nanotubes exist:

(1) Single walled nanotubes (SWNT), made up of only one straight tubular unit [5]. It is fundamental form of carbon nanotube. Single-walled nanotubes are 1 to $2 \mathrm{~nm}$ in diameter and combine to form rope-like structures. The lengths of the ropes are difficult to determine but are approximately $1 \mu \mathrm{m}$ long, with diameters ranging from 10 to $30 \mathrm{~nm}$.

(2) Multi walled nanotubes (MWNT), made up of series of coaxial tubes 0.34 nanometer apart. Any carbon nanotubes can be represented with indices $(\mathrm{n}, \mathrm{m})$ where $\mathrm{n}, \mathrm{m}$ are two integers.

CNTs can either be metallic or semiconducting. Depending on whether the tube is wrapped as a simple cylinder or a tighter helical form, the nanotube material can possess the properties of a semiconductor or a metallic material. The electrical behaviour of CNTs can be determined from the following simple rule which states that the difference between the indices of the tube should be an integral multiple of three. Mathematically, it can be stated that if $(n-m)=3 q$ then it is metallic and if $(n-m) \neq 3 q$ then it is semiconducting where $q$ is an integer. 
Carbon nanotubes also exist in the form of bundles. It can be single walled carbon nanotubes bundle or mixed CNT bundle. SWNT bundle consists of a number of SWNT closely packed within a sheath while mixed CNT bundle consist of both SWNT and MWNT closely packed within the same sheath.

The highest quality carbon nanotube films we have developed achieve roughly $90 \%$ visible-light transmittance and about $200 \Omega / \square$ sheet resistance $R S$. The nanotubes also exhibit other useful properties such as electrical conductivities as high as $100 \Omega^{-1} / \mathrm{cm}^{-1}$, plus high strength, thermal conductivity and flexibility.

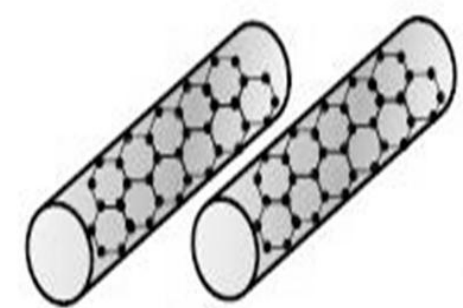

Fig.1.(a) Single walled CNT

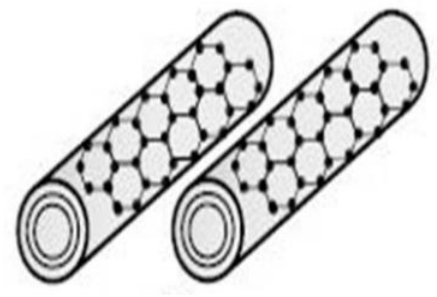

Fig.1.(b) Multi walled CNT

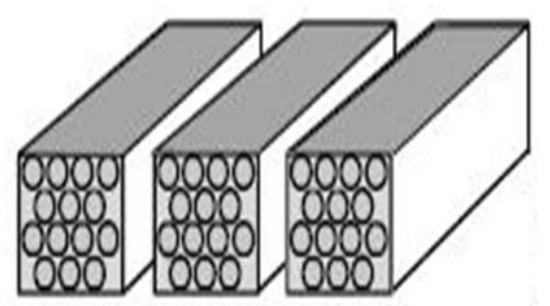

Fig.1.(c) CNT bundle

\subsection{Isolated single-walled carbon nanotube}

\section{Analysis of different CNTs}

\subsubsection{Resistance of Single walled CNT}

The conductance of a carbon nanotube is evaluated using the two-terminal "LANDAUERBUTTIKER" formula [1]. This formula states that, for a 1-D system with $\mathrm{N}$ channels in parallel, the conductance is $\mathrm{G}=\left(\mathrm{Ne}^{2} / \mathrm{h}\right) \mathrm{T}$, where $\mathrm{T}$ is the transmission coefficient for electrons through the sample. Due to spin degeneracy and sub lattice degeneracy of electrons in graphene, each nanotube has four conducting channels in parallel $(\mathrm{N}=4)$. Hence the conductance of a single ballistic single-walled CNT (SWNT) assuming perfect contacts $(\mathrm{T}=1)$, is given by $4 \mathrm{e}^{2} / \mathrm{h}=155 \mu \mathrm{S}$, which yields a resistance of $6.45 \mathrm{k} \Omega$. This is the fundamental resistance associated with a SWCNT, this fundamental resistance $\left(\mathrm{R}_{\mathrm{f}}\right)$ is equally divided between the two contacts on either side of the nanotube. $R_{F}=h / 4 e^{2}=6.45 \mathrm{k} \Omega$, where $h$ is Planck's constant $\left(h=6.626 \times 10^{-34} \mathrm{~J}-\mathrm{S}\right)$, e is the charge of an electron ( $\mathrm{e}=1.602 \times 10^{-19}$ coulomb). The mean free path of electrons (the distance across which no scattering occurs) in a CNT is typically $1000 \mathrm{~nm}$.

For CNT lengths less than $1 \mu \mathrm{m}$, electron transport is essentially ballistic within the nanotube and the resistance is independent of length $(6.45 \mathrm{k} \Omega)$. However, for lengths greater than the mean free path, resistance increases with length as $\mathrm{R}_{\mathrm{CNT}}=\left(\mathrm{h} / 4 \mathrm{e}^{2}\right) \mathrm{L} / \mathrm{Lo}$ where $\mathrm{L}$ is the length of the CNT, Lo is the mean free path $(1000 \mathrm{~nm})$.

\subsubsection{Capacitance of a Single walled CNT}

The capacitance of a CNT arises from two sources. The Electrostatic capacitance $\left(\mathrm{C}_{\mathrm{E}}\right)$ is calculated by treating the CNT as a thin wire, with diameter ' $\mathrm{d}$ ', placed at a distance ' $\mathrm{y}$ ' away from a ground plane, and is given by, $\mathrm{C}_{\mathrm{E}}=(2 \pi \varepsilon) /(\ln (\mathrm{y} / \mathrm{d}))=30 \mathrm{aF} / \mu \mathrm{m}$ [1] where $\varepsilon$ is the permittivity of the dielectric $\left(\varepsilon=\varepsilon_{\mathrm{o}} \varepsilon_{\mathrm{r}}\right), \varepsilon_{\mathrm{o}}=$ $8.854 \times 10^{-18}$. For $22 \mathrm{~nm}$ technology, the relative permittivity $\varepsilon_{\mathrm{r}}=2$.

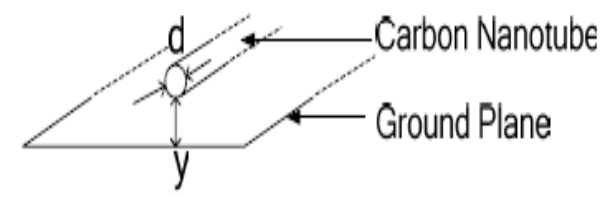

Fig.2. Isolated conductor, with diameter'd', over a ground plane at a distance ' $y$ ' below it.

The Quantum capacitance $\left(\mathrm{C}_{\mathrm{Q}}\right)$ accounts for the quantum electrostatic energy stored in the nanotube when it carries current. Due to the Pauli Exclusion Principle, it is only possible to add electrons into the nanotube at an available quantum state above the Fermi energy level. By equating this energy to an effective capacitance, the expression for the quantum capacitance (per unit length) is obtained as $C_{Q}=2 \mathrm{e}^{2} / \mathrm{hv}_{\mathrm{F}}$ where $\mathrm{h}$ is Planck's constant and $\mathrm{v}_{\mathrm{F}}$ is the Fermi velocity $\left(\mathrm{v}_{\mathrm{F}=} 8 \times 10^{5} \mathrm{~m} / \mathrm{s}\right)$.

As a CNT has four conducting channels, the effective quantum capacitance resulting from four parallel capacitances $C_{Q}$ is given by $4 C_{Q}$. The same effective charge resides on both these capacitances $\left(C_{E}\right.$ and $\left.4 C_{Q}\right)$ when the CNT carries current, as is true for any two capacitances in series. Hence these capacitances appear in series in the effective circuit model.

\subsubsection{Inductance of a Single walled CNT}

The inductance associated with an isolated SWCNT can be calculated from the magnetic field of an isolated current carrying wire some distance away from a ground plane [1]. The magnetic inductance $\left(\mathrm{L}_{\mathrm{M}}\right)$ is as, 
$\mathrm{L}_{\mathrm{M}}=(\mu / 2 \pi)^{*} \ln (\mathrm{y} / \mathrm{d})=1.4 \mathrm{pH} / \mu \mathrm{m}$, Where $\mu$ is the permeability $\left(\mu=\mu_{\mathrm{o}} \mu_{\mathrm{r}}\right), \mu \mathrm{o}=4 \pi \times 10^{-7}$, the relative permeability $\mu_{\mathrm{r}}=1.013 \times 10^{-6}$. In addition to this magnetic inductance $\left(\mathrm{L}_{\mathrm{M}}\right)$, the kinetic inductance is calculated by equating the kinetic energy stored in each conducting channel of the CNT to an effective inductance $L_{K}=(h) /\left(2 \mathrm{e}^{2} v_{F}\right)=$ $16 \mathrm{nH} / \mu \mathrm{m}$.

The four parallel conducting channels in a CNT give rise to an effective kinetic inductance of $\mathrm{L}_{K} / 4$, which gives $4 \mathrm{nH} / \mu \mathrm{m}$. Since $\mathrm{L}_{\mathrm{K}}>>\mathrm{L}_{\mathrm{M}}$, the inclusion of $\mathrm{L}_{\mathrm{K}}$ can have a significant impact on the delay model for interconnects.
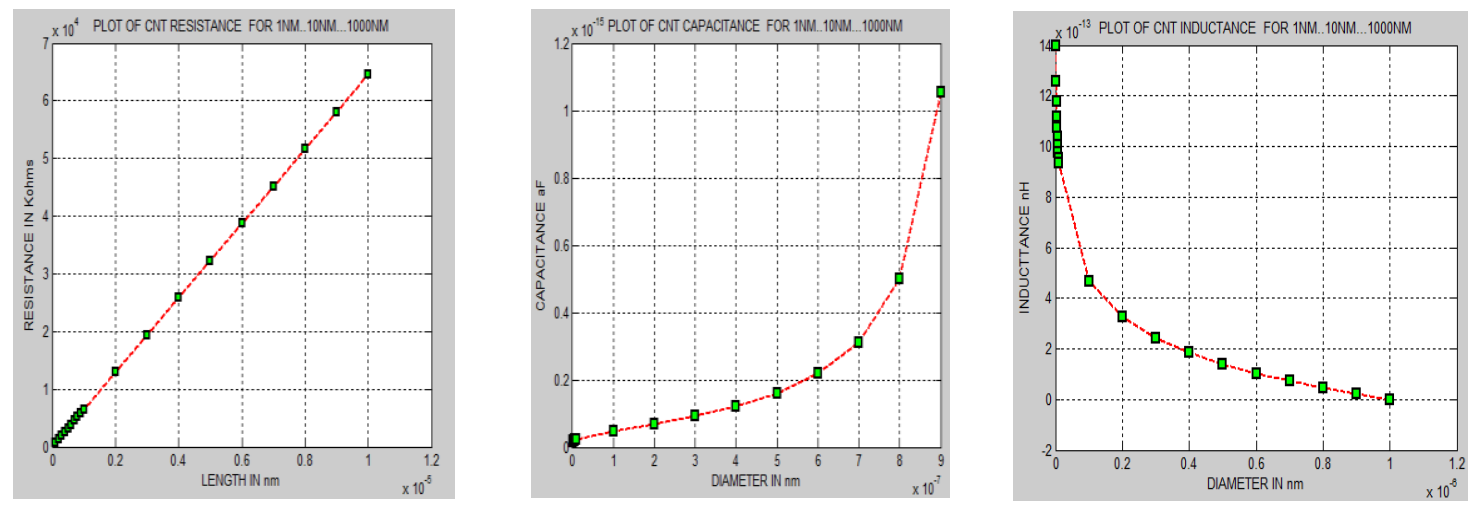

Fig.3.(a) Resistance of SWCNT Fig.3.(b) Capacitance of SWCNT Fig.3.(c) Inductance of Single walled CNT

\subsection{Bundle of $\mathrm{CNT}_{\mathrm{S}}$}

CNT-bundle interconnect is assumed to be composed of hexagonally packed identical metallic singlewalled carbon nanotubes.

\subsubsection{Resistance of a CNT-bundle}

In order to calculate the effective resistance of a CNT-bundle, it is assumed that all CNT's packed into the interconnect structure are metallic and conducting [1]. The fact that it is, in general, difficult to control the conductance properties of all $\mathrm{CNT}_{\mathrm{S}}$ in the bundle is accounted for by considering reduced packing densities. Each CNT is surrounded by six immediate neighbors, their centers uniformly separated by a distance ' $x$ '. The expressions to calculate the number of CNTs in the bundle are $\mathrm{n}_{\mathrm{w}}=\frac{w-d}{x}$ and $\mathrm{n}_{\mathrm{H}=\left[\frac{h-d}{(\sqrt{3} / 2) x}\right]^{+1}}$

where $n_{H}$ is the number of "rows" in the interconnect bundle, $n_{W}$ is the number of "columns", $\mathrm{w}$ and $\mathrm{h}$ are the width and height of the bundle, $n C N T$ is the total number of CNTs, given by $\mathrm{n}_{\mathrm{CNT}=} \mathrm{n}_{\mathrm{W}} \mathrm{n}_{\mathrm{H}}-\frac{n H}{2}$ if $\mathrm{n}_{\mathrm{H}}$ is even and $\mathrm{n}_{\mathrm{CNT}}=\mathrm{n}_{\mathrm{W}} \mathrm{n}_{\mathrm{H}}-\frac{n H-1}{2}$ if $\mathrm{n}_{\mathrm{H}}$ is odd. The CNT-bundle resistance is given by, $\mathrm{R}_{\text {bundle }}=\mathrm{R}_{\text {isolated }} / \mathrm{n}_{\mathrm{CNT}}$.

It must be noted that it is implicit in this formulation that the coupling between adjacent $\mathrm{CNT}_{\mathrm{S}}$ of a bundle is weak. However, this is a fair assumption because it has been shown that there exists a large tunneling resistance $(\sim 2-140 \mathrm{M} \Omega)$ between the $\mathrm{CNT}_{\mathrm{S}}$ forming a bundle.

\subsubsection{Capacitance of a CNT-bundle}

It is observed, as expected, that the $\mathrm{CNT}_{\mathrm{S}}$ completely surrounded by other nanotubes have a very small electrostatic coupling capacitance to ground compared to those along the edges of the bundle (three orders of magnitude smaller) [1].Their contribution to the total electrostatic capacitance is thus neglected. Using these relations, we calculate the electrostatic capacitance contributed by each "edge" CNT based on the corresponding value of $\mathrm{C}_{\mathrm{E}}$. The total electrostatic capacitance of the bundle is given by the sum of the contribution from each of these $\mathrm{CNT}_{\text {s. }}$ i.e., $\mathrm{C}_{\mathrm{E}}=2 \mathrm{C}_{\mathrm{En}}+\left(\mathrm{n}_{\mathrm{w}}-2\right) / 2 * \mathrm{C}_{\mathrm{Ef}}+3\left(\mathrm{n}_{\mathrm{H}}-2\right) / 5 * \mathrm{C}_{\mathrm{En}}$ where $\mathrm{C}_{\mathrm{En}}$ and $\mathrm{C}_{\mathrm{Ef}}$ are the intrinsic plate capacitance for an isolated CNT over a ground plane. $C_{E n}=2 \prod \Sigma / \log (\mathrm{y} / \mathrm{d})$ is calculated assuming the ground plane to be at a distance equal to the separation distance from the "near" adjacent interconnect. $C_{E f}=$ $2 \Pi \Sigma / \log (2 \mathrm{y} / \mathrm{d})$ is calculated assuming the ground plane to be at a distance equal to the separation distance from the "far" adjacent interconnect. Since the quantum capacitances of all the $\mathrm{CNT}_{\mathrm{S}}$ forming a CNT bundle appear in parallel, the effective quantum capacitance of the bundle is the sum of the individual quantum capacitances. $C_{Q \text { bundle }}=C_{Q C N T} * \mathrm{n}_{\mathrm{CNT}}$ where $\mathrm{C}_{\mathrm{Q}}$ is the quantum capacitance of an isolated CNT and $\eta$ is the total number of $\mathrm{CNT}_{S}$ forming the bundle. The effective capacitance $\left(\mathrm{C}_{\mathrm{bundl}}\right)$ of the series combination of a quantum and electrostatic capacitance is given by $1 / C_{\text {bundle }}=1 / C_{Q \text { bundle }}+1 / C_{E \text { bundle }}$. 


\subsubsection{Inductance of a CNT-bundle}

The inductance [1] of a CNT bundle is given by the parallel combination of the inductances corresponding to each CNT forming the bundle, $\mathrm{L}_{\mathrm{bundle}}=\mathrm{L}_{\mathrm{CNT}} / \mathrm{n}_{\mathrm{CNT}}$.
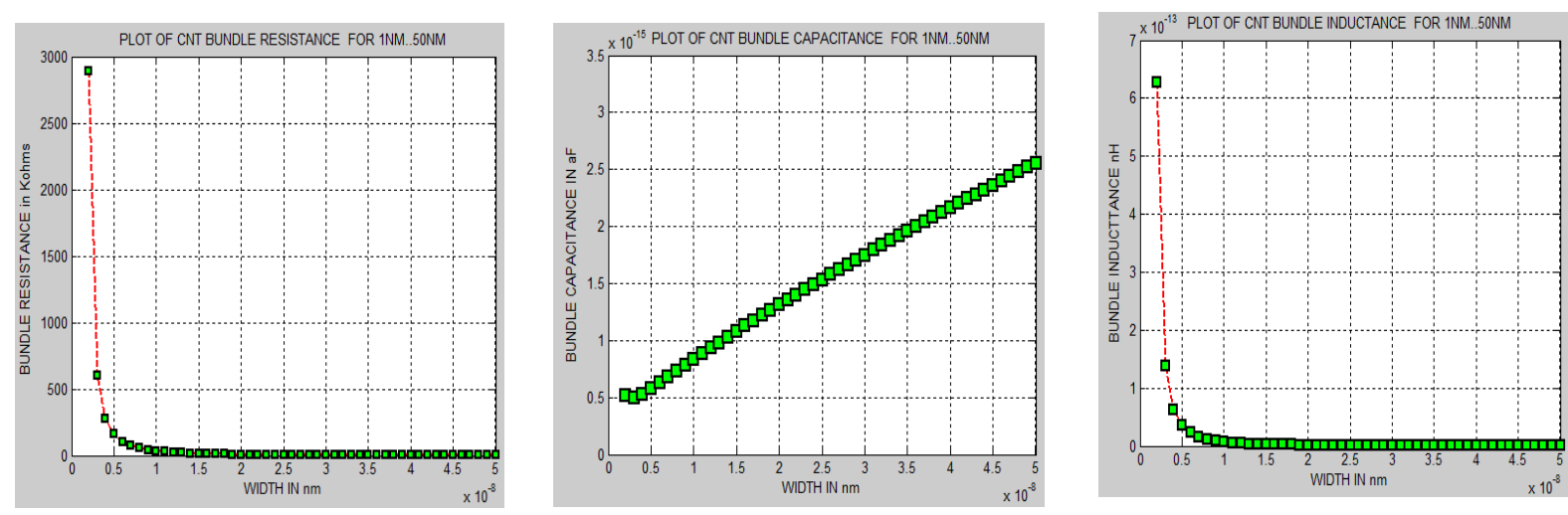

Fig.4.(a) Resistance of CNT bundle Fig.4.(b) Capacitance of CNT bundle Fig.4.(c) Inductance of CNT Bundle

\subsection{Multi walled CNT}

Multi walled nanotubes (MWNT), made up of series of coaxial tubes 0.34 nanometer apart.

\subsubsection{Resistance of multi-walled CNT}

The resistance of a shell consists of three parts: quantum contact resistance RQ, scattering-induced resistance RS, and imperfect contact resistance Rmc [3]. RS only occurs if the length of the nanotube (shell) is larger than the electron MFP. $R Q$ and $R S$ are intrinsic, and $R m c$ is due to fabrication process. The total resistance is determined by $\mathrm{R}=\mathrm{R}_{\mathrm{Q}}+\mathrm{R}_{\mathrm{S}} * \mathrm{~L}=\frac{h}{2 \mathrm{e}^{2} \mathrm{~N}}+\frac{h}{2 \mathrm{e}^{2} \mathrm{~N}} * \frac{\mathrm{L}}{\lambda}$ where $h / 2 \mathrm{e}^{2} \sim 12.9 \mathrm{k} \Omega$, and $L, \lambda$, and $N$ are the length, MFP, and number of conducting channels of the shell, respectively. The imperfect contact resistance $R \mathrm{mc}$ can range from zero to hundreds of kilo-Ohms for different growth processes. $\lambda=D \cdot v F /(\alpha T)$ where $v F$ is the Fermi velocity of CNTs $(\sim 8 \times 105 \mathrm{~m} / \mathrm{s}), \alpha$ is the coefficient of scattering rate, and $T$ is the temperature. Nshell $(D) \approx a \cdot D+b$, where $D$ is the diameter of the shell, $a=0.0612 \mathrm{~nm}^{-1}$, and $b=0.425$.

\subsubsection{Capacitance of multi-walled CNT}

The capacitance of a CNT also has two parts: quantum capacitance $C Q$ and electrostatic capacitance CE [3]. Quantum capacitance per unit length of a shell can be derived as $C Q /$ channel $=2 \times \frac{2 e^{2}}{h v F} \approx 193 \mathrm{aF} / \mu \mathrm{m}$ and $C Q /$ shell $=C Q /$ channel $\times(a \cdot D+b)$.

\subsubsection{Inductance of multi-walled CNT}

For a CNT, there are magnetic inductance and kinetic inductance [3]. The magnetic inductance of each shell is a weak function of the factor $H / D$. For $1<H / D<100$, the magnetic inductance ranges from 0.2 to 1.2 $\mathrm{pH} / \mu \mathrm{m}$, which is much smaller than the kinetic inductance. Hence, the magnetic inductance has been ignored. Kinetic inductance is given by $L K /$ channel $=\frac{h}{2 e^{2} v F} * \frac{1}{2} \approx 8 \mathrm{nH} / \mu \mathrm{m}$ and $L K /$ shell $=L K /$ channel $/(a \cdot D+b)$.

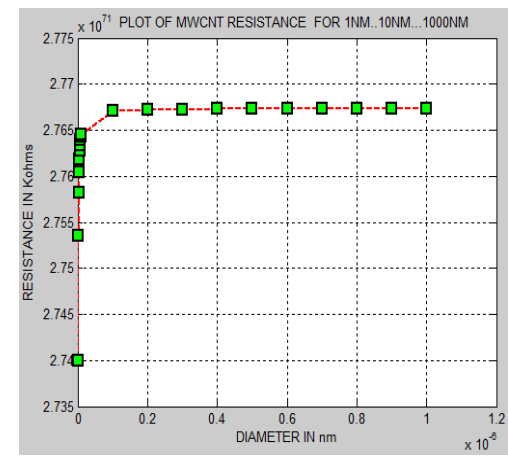

Fig.5.(a) Resistance of MWCNT

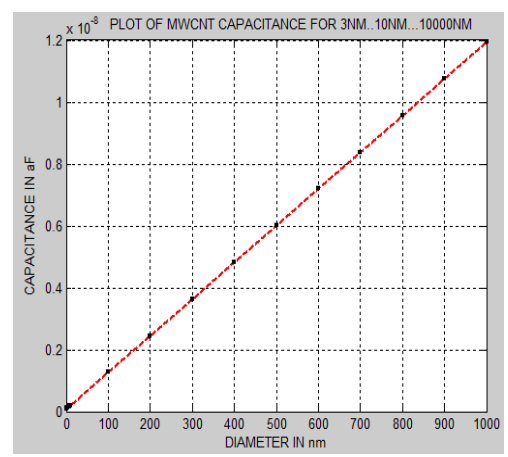

Fig.5.(b) Capacitance of MWCNT

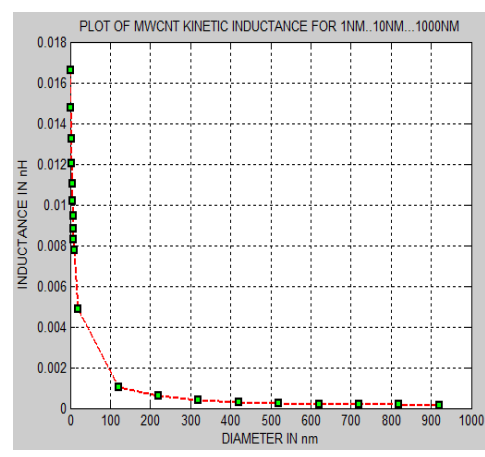

Fig.5.(c) Inductance of MWCNT 


\section{CONCLUSION}

The various results obtained from the matlab simulation reveals the following. For a SWCNT, as the length is reduced, resistance decreases and as the diameter is reduced, capacitance decreases while the inductance increases. For a SWCNT bundle, as the width is reduced, resistance increases, capacitance decreases and inductance increases. For a MWCNT, as the diameter is reduced, resistance decreases, capacitance decreases and inductance increases.

\section{REFERENCES}

[1] Navin Srivastava and Kaustav Banerjee, "Performance Analysis of Carbon Nanotube Interconnects for VLSI Applications".

[2] Arijit Raychowdhury and Kaushik Roy "A Circuit Model for Carbon Nanotube Interconnects: Comparative Study with Cu Interconnects for Scaled Technologies".

[3] Hong Li, Kaustav Banerjee "Circuit Modeling and Performance Analysis of Multi-Walled Carbon Nanotube Interconnects" IEEE JUNE 2008 .

[4] A. Naeemi,et al.," Performance comparison between Carbon Nanotube and Copper Interconnects for Giga scale integration(GSI)", IEEE 2005.

[5] P.L.McEuen,et,al.,'Single-Walled carbon Nanotube Electronics,"IEEE, 2000. 